FGF12 is differentially expressed in the brain metastases of patients with metastatic breast cancer.

Shahan Mamoor, MS ${ }^{1}$

1shahanmamoor@gmail.com

East Islip, NY USA

Metastasis to the brain is a clinical problem in patients with breast cancer ${ }^{1-3}$. We mined published microarray data 4,5 to compare primary and metastatic tumor transcriptomes to discover genes associated with brain metastasis in patients with metastatic breast cancer. We found that the fibroblast growth factor 12, encoded by FGF12, was among the genes whose expression was most different in the brain metastases of patients with metastatic breast cancer as compared to normal breast tissues. FGF12 mRNA expression was significantly higher in brain metastatic tissues as compared to primary tumors of the breast. Upregulation of FGF12 expression may contribute to metastasis of tumor cells from the breast to the brain in humans with metastatic breast cancer.

Keywords: breast cancer, metastasis, brain metastases, central nervous system metastases, fibroblast growth factor 12, FGF12, systems biology of breast cancer, targeted therapeutics in breast cancer. 
One report described a 34\% incidence of central nervous system metastases in patients treated with trastuzumab for breast cancer ${ }^{2}$. More recently, the NEfERT-T clinical trial ${ }^{6}$ which compared administration of either neratinib or trastuzumab in conjunction with paclitaxel demonstrated that in a randomized, controlled setting, in breast cancer patients treated with neratinib, not only was the incidence of central nervous system recurrence significantly lower, the time to central nervous system metastasis was significantly delayed as compared to patients administered trastuzumab. The alarmingly high rate of central nervous system metastasis described, as well as data, both anecdotal ${ }^{2}$ and from a randomized, controlled setting ${ }^{6}$ illustrating that treatment with trastuzumab may be associated with these events demands an enhanced understanding of the transcriptional makeup of brain metastatic tissues to support identification of therapeutic targets, whether they are treatment related or not. We performed a global comparative analysis of the benign breast, primary and metastatic tumors in patients with brain metastatic breast cancer 4,5 . We discovered significant differential and decreased expression of the fibroblast growth Methods

\section{FGF12 is differentially expressed in the brain metastases of patients with brain metastatic breast cancer.}

We identified the fibroblast growth factor 12, encoded by FGF12, as a differentially expressed gene in brain metastases of patients with metastatic breast cancer using a published microarray dataset ${ }^{4}$. When sorting each of the genes expressed in brain metastases based on significance of difference as compared to primary tumors of the breast in patients with breast cancer, FGF12 ranked 23 out of 22575 total transcripts (Table 1), equating to $99.9 \%$ differential expression. Differential expression of FGF12 in the brain metastases of patients with metastatic breast cancer was statistically significant (Table 1; $p=1.77 \mathrm{E}-05$ ).

Identification of differentially expressed genes across datasets in cancer can be difficult and more challenging than when doing so in benign organ tissue and sorted cell populations due to higher levels of heterogeneity in tumors, and differing methods of tumor sampling between laboratories. Thus, we asked 
how gene expression was most significantly different between brain metastases from patients with breast cancer and normal breast tissue as opposed to primary tumors of the breast, by comparing global gene expression profiles of 10 normal breast tissues to 35 brain metastases ${ }^{5}$. Using this strategy, we validated FGF 12 as a differentially expressed gene in the brain metastases of patients with brain metastatic breast cancer $^{5}$ (Table 2). When sorting each of the genes expressed in brain metastases based on significance of difference in expression between brain metastases and normal breast tissues, FGF12 ranked 10819 out of 41093 total transcripts (Table 2), equating to $73.7 \%$ differential expression. Differential expression of FGF12 in brain metastases from patients with brain metastatic breast cancer in this dataset was statistically significant (Table $2 ; p=6.98 \mathrm{E}-03$ ).

Thus, differential expression of FGF12 was conserved both when compared to normal tissues of the breast and to primary tumors of the breast in patients with metastatic breast cancer, in two independent microarray datasets ${ }^{4,5}$.

FGF12 is expressed at higher levels in the brain metastases of patients with brain metastatic breast cancer.

We obtained exact mRNA expression levels for FGF12, in primary tumors of the breast and in brain metastases of patients with brain metastatic breast cancer to determine direction and statistical significance of change in FGF12 expression in brain metastatic tissues. FGF12 was expressed at higher levels in the brain metastases of patients with breast cancer as compared to primary tumors of the breast; this difference was statistically significant (Figure $1 ; p=0.0350$ ).

Thus, by mining published microarray data 4,5 in an unbiased and systematic fashion, we identified the fibroblast growth factor 12, FGF12, as among the genes whose expression was most different, transcriptome-wide, in the brain metastases of patients with breast cancer both when compared to the normal breast and to primary tumors of the breast; FGF12 expression, as compared to primary tumors of the breast, was higher in metastasis to the brain.

\section{Discussion}

We provided evidence here that the fibroblast growth factor 12, encoded by FGF12, is among the genes whose expression is most different in the brain metastases of patients with brain metastatic breast cancer. Evaluating phenotypic consequences of FGF12 genetic depletion in mouse models of metastatic breast cancer on metastasis to the central nervous system is merited. 


\section{References}

1. Lin, N.U., Amiri-Kordestani, L., Palmieri, D., Liewehr, D.J. and Steeg, P.S., 2013. CNS metastases in breast cancer: old challenge, new frontiers.

2. Bendell, J.C., Domchek, S.M., Burstein, H.J., Harris, L., Younger, J., Kuter, I., Bunnell, C., Rue, M., Gelman, R. and Winer, E., 2003. Central nervous system metastases in women who receive trastuzumab-based therapy for metastatic breast carcinoma. Cancer, 97(12), pp.2972-2977.

3. Tsukada, Y., Fouad, A., Pickren, J.W. and Lane, W.W., 1983. Central nervous system metastasis from breast carcinoma autopsy study. Cancer, 52(12), pp.2349-2354.

4. Harrell, J.C., Prat, A., Parker, J.S., Fan, C., He, X., Carey, L., Anders, C., Ewend, M. and Perou, C.M., 2012. Genomic analysis identifies unique signatures predictive of brain, lung, and liver relapse. Breast cancer research and treatment, 132(2), pp.523-535.

5. Salhia, B., Kiefer, J., Ross, J.T., Metapally, R., Martinez, R.A., Johnson, K.N., DiPerna, D.M., Paquette, K.M., Jung, S., Nasser, S. and Wallstrom, G., 2014. Integrated genomic and epigenomic analysis of breast cancer brain metastasis. PloS one, $9(1)$, p.e85448.

6. Awada, A., Colomer, R., Inoue, K., Bondarenko, I., Badwe, R.A., Demetriou, G., Lee, S.C., Mehta, A.O., Kim, S.B., Bachelot, T. and Goswami, C., 2016. Neratinib plus paclitaxel vs trastuzumab plus paclitaxel in previously untreated metastatic ERBB2-positive breast cancer: the NEfERT-T randomized clinical trial. JAMA oncology, 2(12), pp.1557-1564. 
Table 1: FGF12 is differentially expressed in brain metastases of patients with metastatic breast cancer when compared to primary tumors of the breast.

Rank of differential expression, probe ID, $p$-value with respect to differential expression, $t$, a moderated tstatistic, B, the log-odds of differential expression between the two groups compared, gene and gene name are listed in this chart.

PAGE 5 
Table 2: FGF12 is differentially expressed in brain metastases in patients with metastatic breast cancer when compared to normal breast tissues.

Rank of differential expression, probe ID, $p$-value with respect to differential expression, $t$, a moderated tstatistic, B, the log-odds of differential expression between the two groups compared, gene and gene name are listed in this chart.

\section{PAGE 6}




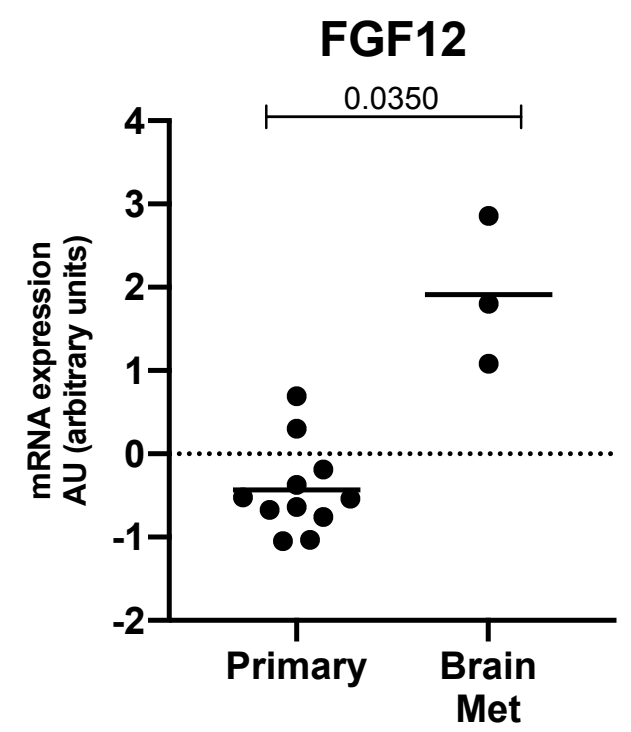

Figure 1: FGF12 is expressed at significantly higher levels in the brain metastases of patients with brain metastatic breast cancer as compared to primary tumors of the breast.

The mRNA expression level of FGF12 in primary tumors of the breast (left) and in the brain metastases of brain metastatic breast cancer patients (right) is graphically represented here with mean FGF12 levels marked and the result of a statistical test evaluating difference in mRNA expression level between brain metastases and primary tumors of the breast, a $p$-value, listed above. 